\title{
Eficacia antibacteriana de dos enjuages bucales (Triclosan y cloruro de cetilpiridinio) sobre streptococos orales
}

Enrique Estela ${ }^{1}$

Cecilia Ponce ${ }^{2}$

\section{Resumen}

El objetivo de este trabajo fue evaluar la actividad antimicrobiana de dos enjuagues bucales sobre streptococcus orales. Material y métodos: La investigación realizada fue de tipo clínico, prospectivo y longitudinal en un total de 45 pacientes de ambos sexos cuyas edades oscilaron entre 21 y 35 años de edad. Los pacientes fueron asignados aleatoriamente en tres grupos; el primero que recibió colutorios de cloruro de cetilpiridinio, el segundo que recibió colutorios de triclosán y el tercero que fue consignado como grupo-control (colutorios con agua). Previo al uso del enjuague bucal se realizó una encuesta a la totalidad de pacientes con respecto a los hábitos básicos de higiene bucal y un odontograma en el que se determinó el riesgo de caries de los sujetos incluidos en el estudio. Así mismo se realizó un recuento de colonias de streptococos orales en una muestra de saliva inicial de cada paciente. Después del uso del colutorio respec- tivo se realizó un nuevo recuento de colonias de streptococos, cuyos resultados fueron contrastados con la muestra inicial.

Los resultados mostraron que el uso de triclosán o cloruro de cetilpiridinio reducen significativamente el recuento de colonias de streptococos orales. La reducción media en el recuento de streptococos fue 146,200 UFC/ml en los pacientes que usaron triclosán y 113,670 UFC/ml en los pacientes que utilizaron cloruro de cetilpiridinio. Además, se halló una asociación significativa entre el tipo de saliva, el número de superficies de caries y el recuento inicial de colonias de streptococos. $(\mathrm{p}<0.05)$. Conclusiones: Se concluyó en la verificación experimental acerca de la mayor eficacia que presentó el colutorio de triclosán frente al colutorio de cloruro de cetilpiridinio.

Palabras Clave: antiséptico bucal, triclosán, cetilpiridinio, streptococos.

Artigo Original

\section{Eficácia antibacteriana de anti-sépticos bucais (Triclosan e cetil piridínio) em streptococcus orais}

\section{Resumo}

O objetivo deste trabalho foi avaliar a atividade antimicrobiana de dos anti-sépticos bucais so- bre streptococcus orais. Método: A amostra foi constituída de 45 individuos entre 21 e 35 anos. Eles foram designaram em tres grupos, cloreto

\footnotetext{
${ }^{1}$ Cirujano Dentista FO USMP, Diplomado en Rehabilitación Oral FE UCSUR

${ }^{2}$ Cirujano Dentista FO USMP, Diplomado en Ortodoncia FE UCSUR
} 
de cetilpiridinio, triclosan e control. Prévio nos enxaguatorios uma enquête foi feita a todos os pacientes com respeito a seus hábitos de higiene bucal e odontograma para determinar seu risco de cárie. Una primeira amostra foi obtida de cada paciente e uma segunda amostra depois o enxaguatorios. Os resultados obtidos comprovaram que substâncias anti-sépticas podem constituir-se em opção complementar para reduzir significativamente a presença de colonias de streptococcus orais. A redução media no reconto de treptococcus orais foi 146,200 UFC/ml nos pacientes que usaram triclosan e 113,670 UFC / $\mathrm{ml}$ nos pacientes que usaram cloreto de cetilpiridinio. Conclusão: Além disso, encontro-se uma associação significante entre e número de superficie de cárie e o reconto inicial de colonias de streptococcus.

Palavras-chave: Antissépticos bucais,triclosan,c etilpiridinio, streptococcus.

\section{Original article}

\section{Antibacterial efficacy of two mouthwashes (Triclosan and Cetylpyridinium Chloride) against oral Streptococci}

\section{Abstract}

The aim of this research was to evaluate the antimicrobial activity of two oral antiseptics against oral streptococcus. A clinical, prospective and longitudinal study was carried out in a sample of 45 patients. Methods: Male and female subjects from 21 to 35 years old were included in this research. They were randomly selected in three groups; group 1 (Cetylpyridinium Chloride), group 2 (Triclosan), and group 3 (Water) as a control. A questionnaire regarding daily oral hygiene habits and an odontogram to determine caries risk was performed before using any mouthrinse. Likewise, saliva was collected with the aim to calculate the initial CFU/ml of oral streptococci. Eventually, saliva samples were collected to count post-rinse $\mathrm{CFU} / \mathrm{ml}$ of oral streptococci and contrast it with the initial sample. Final results showed that the use of either triclosan or cetylpyridinium chloride reduce significantly the CFU/ml of oral streptococci. The average reduction on the triclosan group was 146,200 CFU/ml whereas it was 113,670 $\mathrm{CFU} / \mathrm{ml}$ on cetylpyridinium chloride group. Furthermore, a significant association amongst the sort of saliva, the number of decay surfaces, and the initial CFU/ml of oral streptococci was found. $(p<0.05)$ It was concluded under experimental verification that triclosan is more effective than cetylpyridinium chloride. Therefore, as a result a statistical significance between the two mouthwashes was demonstrated.

Key Words: Mouthrinse, triclosan, cetylpyridinium, streptococci.

\section{Introducción}

Actualmente sabemos que el tratamiento restaurador de las lesiones de caries no mejora la salud bucal de las personas sino que sólo limita y repara un daño ya producido por la enfermedad. $(4,10)$ Por lo tanto el tratamiento debe ser enfocado primero a eliminar o disminuir los niveles de microorganismos que causan la caries y luego restaurar los tejidos dañados por ésta. (4) 
Dentro de los mecanismos encargados de disminuir o suprimir la microflora oral encontramos los enjuagues bucales. (9) En nuestro medio, existe una amplia difusión acerca del uso diario de colutorios o antisépticos bucales. En las últimas décadas, la industria farmacéutica ha irrumpido ampliamente en odontología a través de la introducción al mercado de un sinnúmero de enjuagatorios bucales. Debido a dicha difusión, los colutorios han dejado de ser aplicados luego de una rigurosa prescripción médicoodontológica para ser comercializados como productos populares expendidos incluso en supermercados. $(11,12)$

Esta realidad ha llevado al odontólogo a investigar clínicamente la eficacia de distintos colutorios como el triclosán y el cloruro de cetilpiridinio. El Triclosan es un derivado fenólico no iónico de baja toxicidad y de amplio espectro, que ha sido incorporado exitosamente en enjuagues bucales. La evidencia científica disponible muestra que la formula activa de triclosan en enjuagues bucales puede influenciar la actividad microbiana y consecuentemente su eficiencia clínica. Solo como colutorio al 0,2\% tiene un efecto inhibitorio moderado de la placa y una sustantividad antimicrobiana de alrededor cinco horas. $(3,8)$ Por otro lado el cloruro de cetilpiridinio es un compuesto de amonio cuaternario catiónico que reduce la placa en un 35\%. Este compuesto es de eficacia moderada y se elimina rápidamente de las superficies bucales. (4)

\section{Material y Métodos}

En este estudio se incluyeron 45 pacientes cuyas edades oscilaron entre 21 y 35 años. La unidad de estudio estuvo constituida por la saliva presente en la cavidad bucal de los pacientes incluidos en el estudio.
Se dividió la muestra total en 3 grupos, un primer grupo constituido por 15 pacientes, los cuales procedieron al enjuague con triclosan, un segundo grupo constituido también por 15 pacientes, los cuales procedieron a enjuagarse con cloruro de cetilpiridinio, y un tercer grupo que sirvió de control, formado por 15 pacientes los cuales realizaron enjuagues con agua.

Los pacientes fueron seleccionados de acuerdo a los siguientes criterios:

Pacientes con buen estado de salud general; que presentaban actividad cariogénica elevada y con un alto riesgo a nuevas lesiones cariosas; excluyendo aquellos con terapia farmacológica actual o 90 días anteriores a la toma de muestra, además de aquellos que usaban prótesis removibles, fijas o aparatos de ortodoncia.

Se realizó un examen estomatológico completo incluyendo odontograma (para seleccionar a los de alto riesgo de caries de acuerdo con la clasificación de la ADA) e índice de higiene oral de Silness y Löe, al total de los pacientes y se asignaron aleatoriamente al grupo de control y a los de experimentación.

El procedimiento desarrollado con cada paciente fue el siguiente:

a) Antes de empezar el procedimiento el paciente depositó $5 \mathrm{ml}$ de saliva no estimulada como primera muestra.

b) Seguidamente se practicó el enjuague con $10 \mathrm{ml}$ de acuerdo a lo establecido con los enjuagues bucales puros durante 30 segundos.

c) Luego de transcurridos 60 segundos de haber eliminado el enjuague, se procedió a tomar nuevamente la muestra, en la cual el paciente depositó $5 \mathrm{ml}$ de saliva no estimulada como segunda muestra. 
Luego de recolectada la muestra, en laboratorio se realizó la dilución de $1 / 10$, es decir $1 \mathrm{ml}$ de saliva en $9 \mathrm{ml}$ de suero fisiológico. Se procedió a la siembra de la muestra (10ul), en agar mitis salivarius, incubados a $36+1^{\circ} \mathrm{C}$ por 48 horas en anaerobiosis. El recuento total del número de colonias fue multiplicado por 1000 para determinar el total de colonias de streptococos orales.

\section{Análisis Estadístico}

Todos los datos registrados provinieron de la comparación entre los tres grupos (uno de control y dos de experimentación) de pacientes pre y post enjuague. Se estimó la reducción de la población microbiana, los cuales fueron expresados en términos porcentuales, almacenados en un programa de base de datos y tabulados en el programa de SPSS 8.0. Posteriormente, con las diferencias obtenidas se realizaron los siguien- tes análisis estadísticos: análisis porcentual (\%), chicuadrado, media aritmética y desviación estándar para determinar si las diferencias observadas eran estadísticamente significativas.

\section{Resultados}

En la tabla 1 se observan los valores de la estadística descriptiva de las variables en estudio. Se aprecia una diferencia notable de la media aritmética entre el recuento de colonias antes y después del enjuague.

De acuerdo con la Tabla 2, es importante destacar que la totalidad de pacientes evaluados manifestó ingerir "snacks" (alimentos variados entre comidas). También fue significativo el hecho que la amplia mayoría reconoció ingerir al menos 3 "snacks" al día $(20$ casos $=44.4 \%)$. Asimismo en la Tabla 3, apreciamos el recuento de

Tabla 1. Estadística descriptiva de las variables estudiadas.

\begin{tabular}{|l|r|r|r|r|r|}
\hline & RANGO & MINIMO & MAXIMO & MEDIA & $\begin{array}{c}\text { DESV } \\
\text { ESTANDAR }\end{array}$ \\
\hline EDAD & 14 & 21 & 35 & 29.84 & 5.12 \\
$\begin{array}{l}\text { N॰ SUPERFICIES DE } \\
\text { CARIES }\end{array}$ & 11 & 5 & 16 & 10.20 & 3.00 \\
$\begin{array}{l}\text { RECUENTO DE } \\
\text { COLONIAS (PRE) } \\
\begin{array}{l}\text { RECUENTO DE } \\
\text { COLONIAS (POST) }\end{array}\end{array} \quad 188$ & 112 & 300 & 286.18 & 40.31 \\
\hline
\end{tabular}

Tabla 2. Distribución de pacientes en los tres grupos evaluados según frecuencia de "snacks" al día.

\begin{tabular}{|lll|r|r|r|r|}
\hline & & & \multicolumn{2}{|c|}{ TIPO DE COLUTORIOS } & \multirow{2}{*}{} \\
\cline { 3 - 7 } & & & & & & \multirow{2}{*}{ Total } \\
\hline$N^{\circ}$ SNACKS & 1 & $N^{\circ}$ & 5 & 2 & 4 & 11 \\
AL DIA & $\%$ & $33.3 \%$ & $13.3 \%$ & $26.7 \%$ & $24.4 \%$ \\
\cline { 2 - 6 } & 2 & $N^{\circ}$ & 5 & 7 & 2 & 14 \\
& & $\%$ & $33.3 \%$ & $46.7 \%$ & $13.3 \%$ & $31.1 \%$ \\
\cline { 2 - 6 } & $>3$ & $N^{\circ}$ & 5 & 6 & 9 & 20 \\
& & $\%$ & $15.3 \%$ & $40.0 \%$ & $60.0 \%$ & $44.4 \%$ \\
\hline Total & & $N^{\circ}$ & $100.0 \%$ & $100.0 \%$ & $100.0 \%$ & $100.0 \%$ \\
\hline
\end{tabular}


Tabla 3. Recuento de colonias antes del enjuague según frecuencia de "snacks".

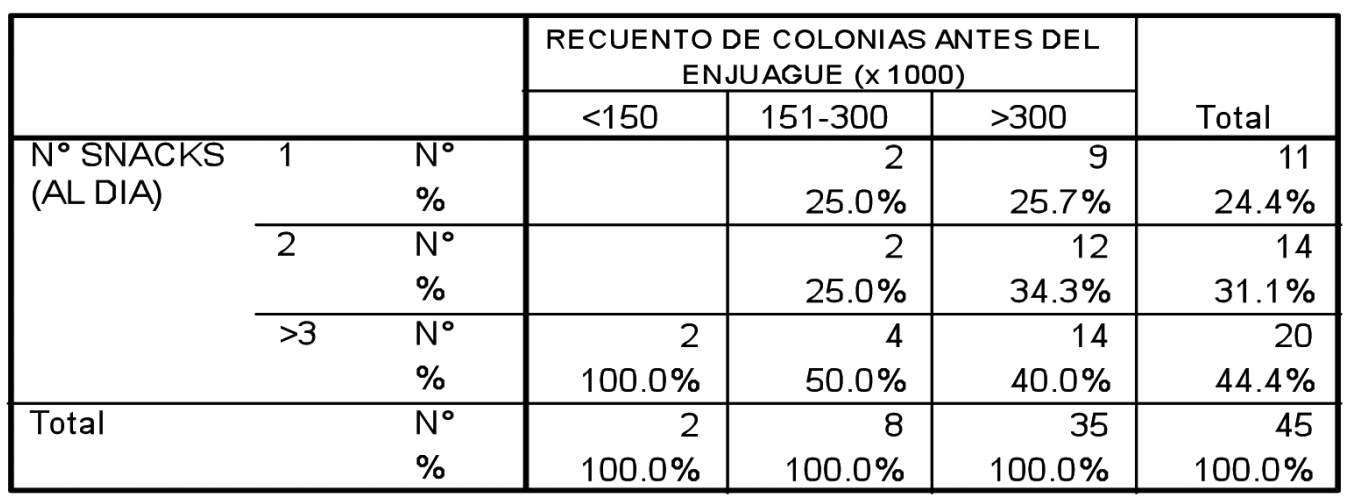

Tabla 4. Distribución de pacientes en los tres grupos evaluados según tipo de saliva.

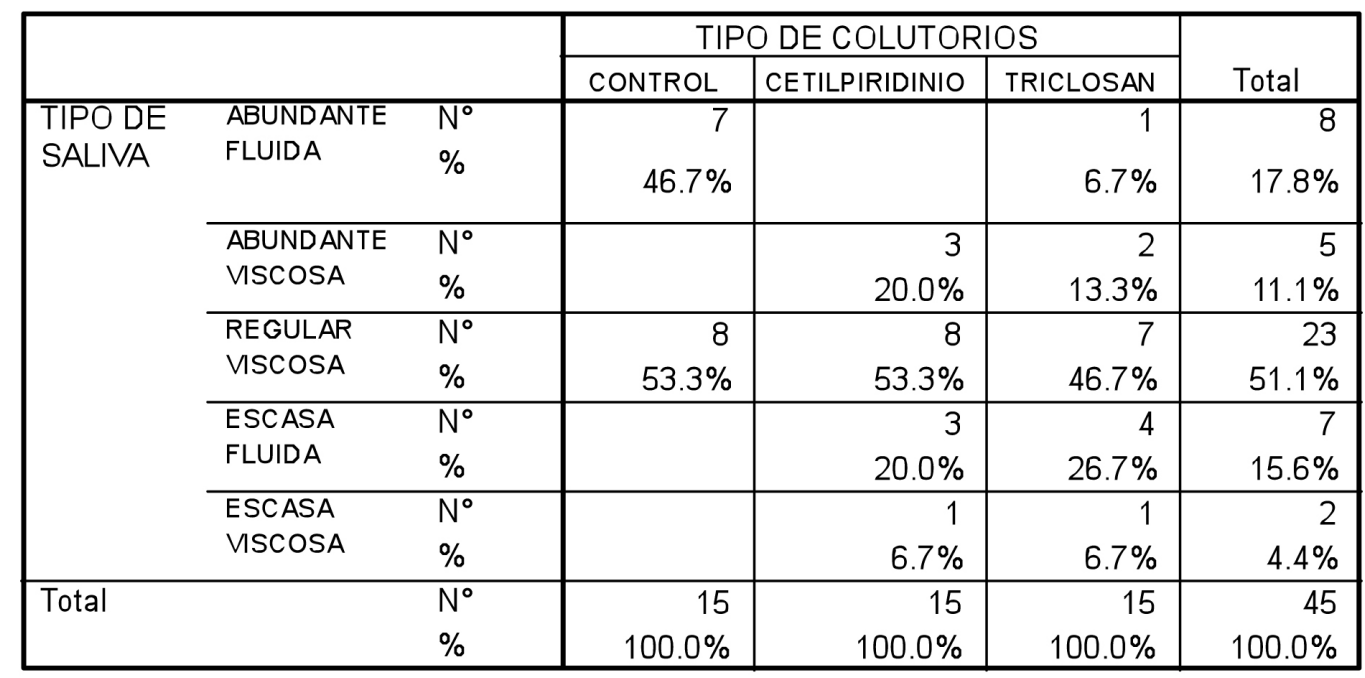

Tabla 5. Recuento de colonias antes del enjuague según el tipo de saliva.

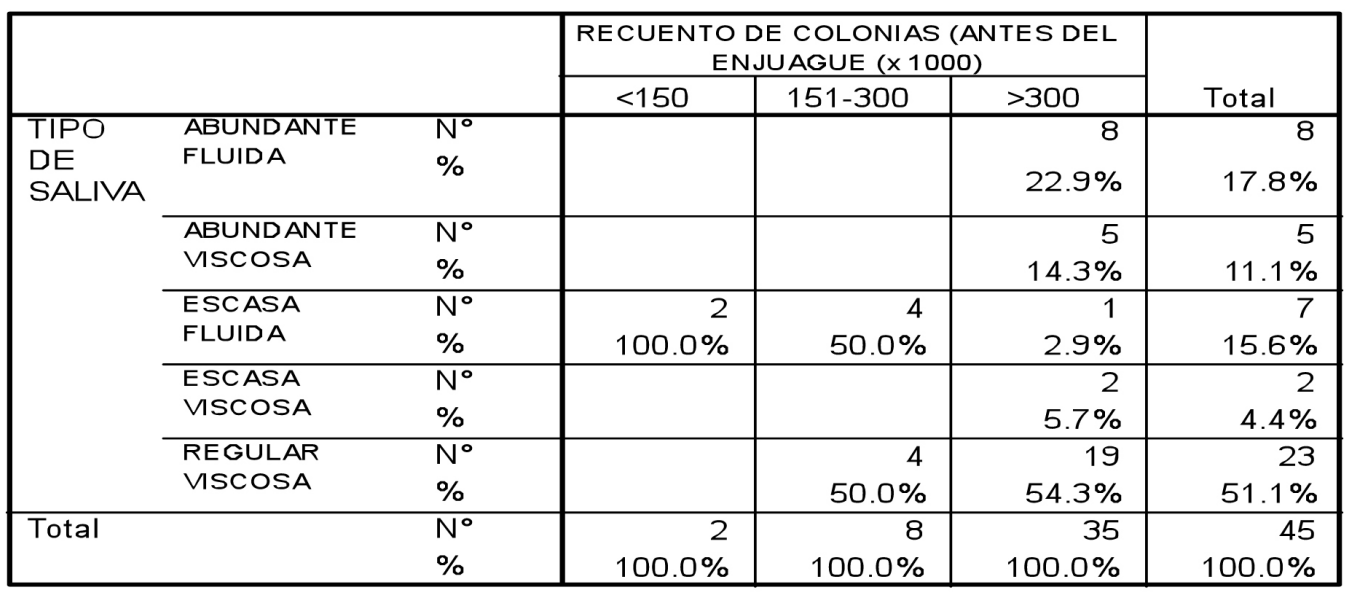

colonias antes del enjuague según frecuencia de "snacks", el resultado fue que no se halló una relación estadísticamente significativa entre el recuento inicial de colonias de streptococos y el número de "snacks" ingeridos entre las comidas.
Por otro lado, la tabla 4, muestra que la mayoría de pacientes evaluados presentaron una saliva considerada clínicamente como regular-viscosa $(23$ casos en general $=51.1 \%$ del total de la muestra). Sólo se encontraron cinco casos por- 
tadores de saliva calificada clínicamente como abundante-viscosa (11.1\%). El recuento de colonias antes del enjuague según el tipo de saliva, es observado en la Tabla 5, así, los pacientes que presentaron inicialmente mayor recuento en el número de colonias, la mayoría (19 casos = $54.3 \%$ ) correspondió a sujetos con saliva de tipo regular-viscosa. Es decir al presentar este tipo de saliva vamos a encontrar mayor número de microorganismos en el recuento.

En relación al riesgo de caries, la Tabla 6 nos permite observar una similitud en la distribución de pacientes según el número de superficies cariadas. En general, la mayoría de pacientes presentaron entre 9-12 superficies de caries dental (24 casos $=53.3 \%$ ). Así mismo, de acuerdo con la Tabla 7 , el recuento de colonias antes del enjuague según el número de superficies de caries, tuvo una asociación estadísticamente significativa entre el recuento inicial de colonias de streptococos (antes de enjuague) y el número de superficies de caries $(\mathrm{p}<0.05)$. esto representa un hallazgo importante desde el punto de vista clínico.

La mayoría de casos evaluados, independientemente del tipo de colutorios utilizados, presentaron un Índice de higiene oral de grado 2 (36 casos $=80 \%$ del total de la muestra). Dicha tendencia se observó en los distintos grupos experimentales, además existe una relación estadísticamente significativa entre el recuento inicial de colonias y el índice de higiene oral, podemos apreciar que en aquellos pacientes que se determinó un recuento mayor a 300 colonias, presentaron un índice de higiene oral de grado $3(80.0 \%)$, como es observado en las tablas 8 y 9 respectivamente.

Tabla 6. Distribución de pacientes en los tres grupos evaluados según número de superficies de caries.

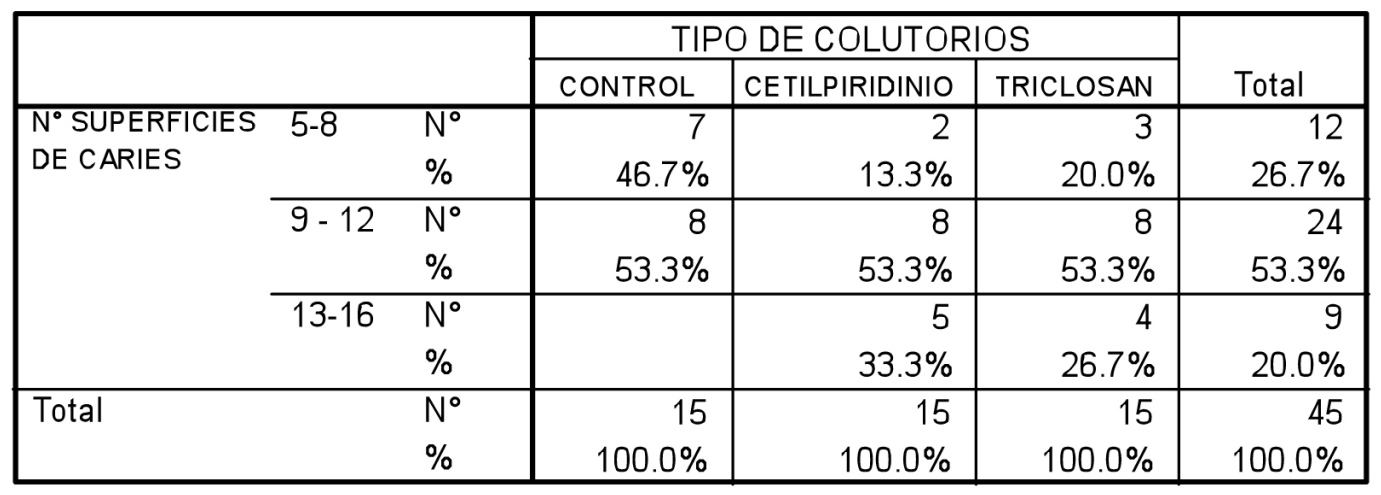

Tabla 7. Recuento de colonias antes del enjuague según el número de superficies de caries.

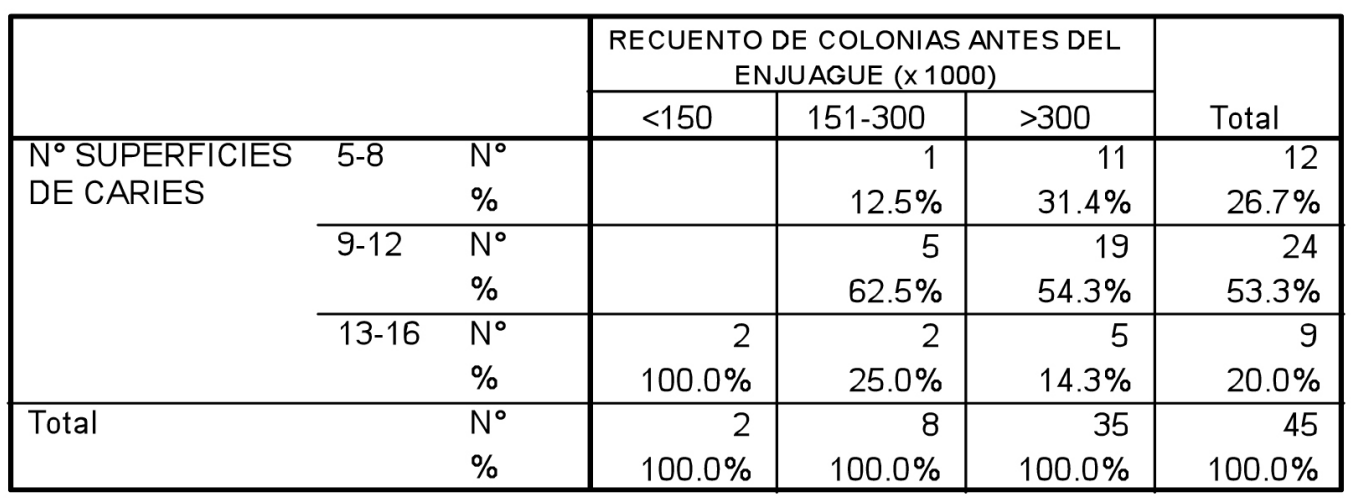


Tabla 8. Distribución de pacientes en los tres grupos evaluados según índice de higiene Oral simplificado (Silness-Lóe).

\begin{tabular}{|c|c|c|c|c|c|c|}
\hline & & & \multicolumn{3}{|c|}{ TIPO DE COLUTORIOS } & \multirow[b]{2}{*}{ Total } \\
\hline & & & CONTROL & CETILPIRIDINIO & TRICLOSAN & \\
\hline \multirow{6}{*}{$\begin{array}{l}\text { INDICE } \\
\text { SILNESS- } \\
\text { LOE }\end{array}$} & 1 & $\mathrm{~N}^{\circ}$ & 3 & & & 3 \\
\hline & & $\%$ & $20.0 \%$ & & & $6.7 \%$ \\
\hline & 2 & $\mathrm{~N}^{\circ}$ & 12 & 12 & 12 & 36 \\
\hline & & $\%$ & $80.0 \%$ & $80.0 \%$ & $80.0 \%$ & $80.0 \%$ \\
\hline & 3 & $\mathrm{~N}^{\circ}$ & & 3 & 3 & 6 \\
\hline & & $\%$ & & $20.0 \%$ & $20.0 \%$ & $13.3 \%$ \\
\hline \multirow{2}{*}{\multicolumn{2}{|c|}{ Total }} & $\mathrm{N}^{\circ}$ & 15 & 15 & 15 & 45 \\
\hline & & $\%$ & $100.0 \%$ & $100.0 \%$ & $100.0 \%$ & $100.0 \%$ \\
\hline
\end{tabular}

Tabla 9. Recuento inicial de colonias según el índice de higiene oral.

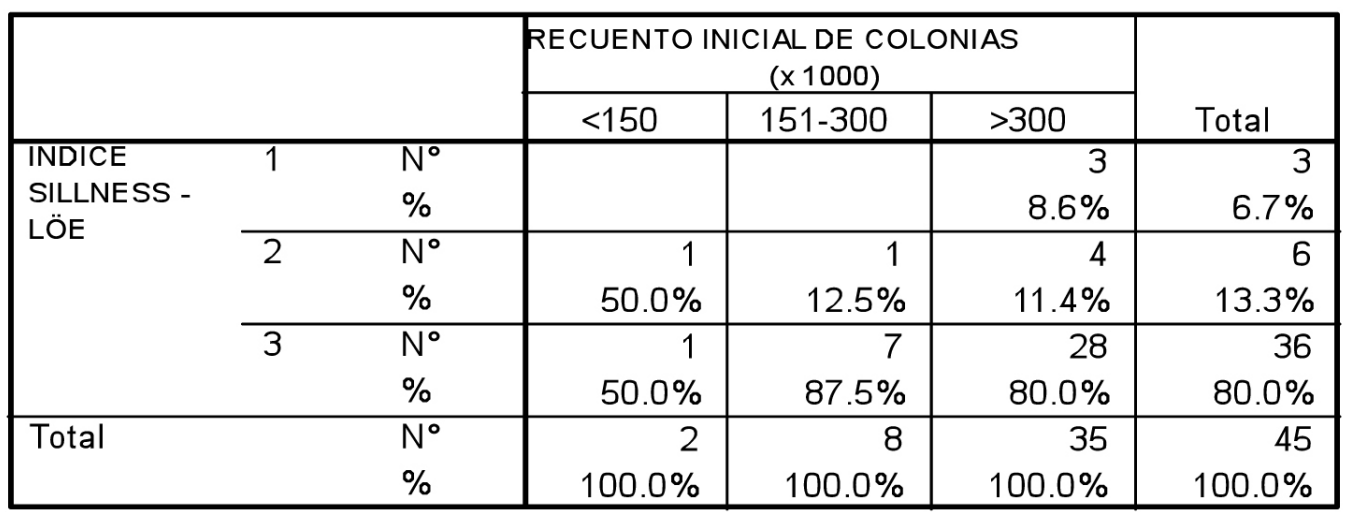

Tabla 10. Tipo de colutorios aplicados según $n^{\circ}$ de colonias recolectadas (antes del enjuague).

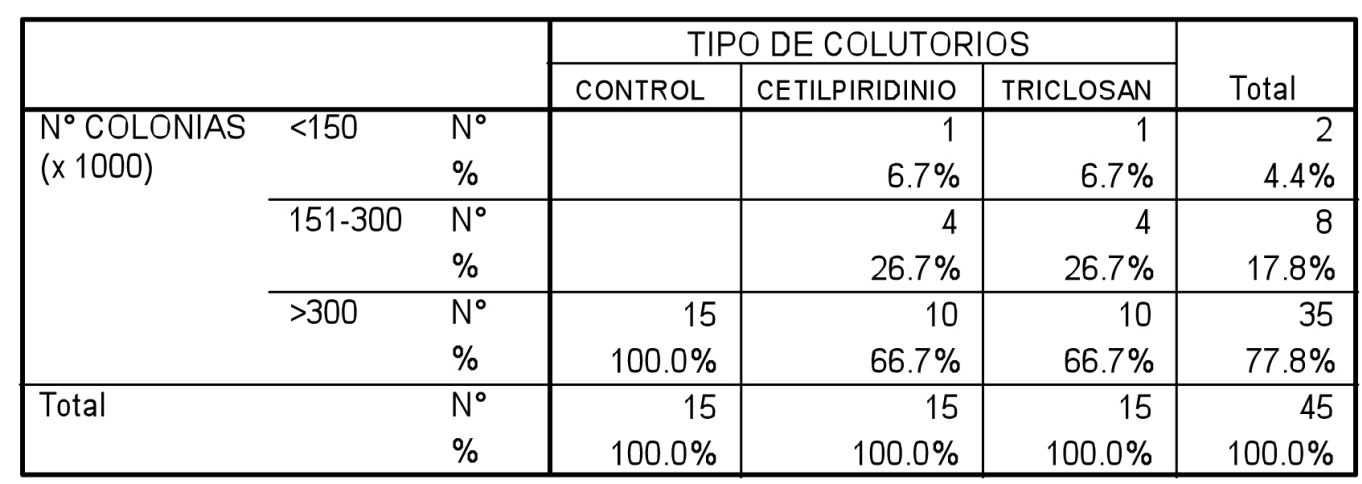

La Tabla 10, muestra la distribución de colutorios aplicados y el número de colonias recolectadas (antes del enjuague); como era de esperar, no se halló relación significativa entre el tipo de colutorio utilizado con el recuento inicial de colonias. Hubo similar distribución en el recuento del número de colonias antes de la aplicación del colutorio (triclosan, cloruro de cetilpiridinio y agua). La mayoría de pacientes presentaron un recuento inicial mayor de 300,000 colonias.

La Tabla 11, sin embargo nos permite observar la relación entre el tipo de colutorio aplicado y el número de colonias recolectadas (después del enjuagatorio), en la cual se halló una asociación estadísticamente significativa entre el recuento 
final de colonias y el tipo de colutorio aplicado. De manera general se observó una diferencia significativa entre aquellos pacientes que recibieron algún colutorio y el grupo control; es decir, que todos los pacientes pertenecientes al grupo-control presentaron más de 150,000 colonias. También se observó que aquellos pacientes que recibieron tratamiento de colutorios con Triclosán presentaron recuentos de colonias menores de 150,000 (9 casos $=60 \%$ ), a diferencia de aquellos que recibieron cetilpiridinio (8 casos $=53.3 \%$ ).
En la Tabla 12, después de determinar la diferencia entre ambos recuentos de colonias para cada una de las muestras, se halló mayor variación en el grupo de pacientes que realizaron colutorios con triclosán. Esto se deduce de la mayor frecuencia de pacientes cuyos recuentos de colonias variaron más (variación mayor de 200,000 colonias antes y después del colutorio). Esta variación fue estadísticamente significativa $(\mathrm{p}<0.05)$.

La variación entre el recuento inicial y final de colonias (antes y después de la aplicación del

Tabla 11. Tipo de colutorios aplicados según $n^{\circ}$ de colonias recolectadas (después del enjuagatorio).

\begin{tabular}{|c|c|c|c|c|c|c|}
\hline & \multicolumn{3}{|c|}{ TIPO DE COLUTORIOS } & \multirow[b]{2}{*}{ Total } \\
\hline & & & CONTROL & CETILPIRIDINIO & TRICLOSAN & \\
\hline \multirow{6}{*}{$\begin{array}{l}\mathrm{N}^{\circ} \mathrm{COLONIAS} \\
(\mathrm{x} 1000)\end{array}$} & $<150$ & $\mathrm{~N}^{0}$ & & 8 & 9 & 17 \\
\hline & & $\%$ & & $53.3 \%$ & $60.0 \%$ & $37.8 \%$ \\
\hline & $151-300$ & $\mathrm{~N}^{\circ}$ & 6 & 4 & 5 & 15 \\
\hline & & $\%$ & $40.0 \%$ & $26.7 \%$ & $33.3 \%$ & $33.3 \%$ \\
\hline & $>300$ & $N^{\circ}$ & 9 & 3 & 1 & 13 \\
\hline & & $\%$ & $60.0 \%$ & $20.0 \%$ & $6.7 \%$ & $28.9 \%$ \\
\hline \multirow[t]{2}{*}{ Total } & & $\mathrm{N}^{\circ}$ & 15 & 15 & 15 & 45 \\
\hline & & $\%$ & $100.0 \%$ & $100.0 \%$ & $100.0 \%$ & $100.0 \%$ \\
\hline
\end{tabular}

Tabla 12. Tipo de colutorios aplicados según variación en el recuento de colonias (X1000).

\begin{tabular}{|c|c|c|c|c|c|c|}
\hline & & & \multicolumn{3}{|c|}{ TIPO DE COLUTORIOS } & \multirow[b]{2}{*}{ Total } \\
\hline & & & CONTROL & CETILPIRIDINIO & TRICLOSAN & \\
\hline \multirow{6}{*}{$\begin{array}{l}\text { VARIACION } N^{\circ} \\
\text { COLONIAS } \\
(\times 1000)\end{array}$} & $0-100$ & $\overline{N^{0}}$ & 15 & 8 & 5 & 28 \\
\hline & & $\%$ & $100.0 \%$ & $53.3 \%$ & $33.3 \%$ & $62.2 \%$ \\
\hline & $101-200$ & $\mathrm{~N}^{\circ}$ & & 3 & 7 & 10 \\
\hline & & $\%$ & & $20.0 \%$ & $46.7 \%$ & $22.2 \%$ \\
\hline & $>200$ & $\mathrm{~N}^{\circ}$ & & 4 & 3 & 7 \\
\hline & & $\%$ & & $26.7 \%$ & $20.0 \%$ & $15.6 \%$ \\
\hline \multirow[t]{2}{*}{ Total } & & $\mathrm{N}^{\circ}$ & 15 & 15 & 15 & 45 \\
\hline & & $\%$ & $100.0 \%$ & $100.0 \%$ & $100.0 \%$ & $100.0 \%$ \\
\hline
\end{tabular}

Tabla 13. Variación en recolección de colonias (Pre-Post).

\begin{tabular}{|l|r|r|r|}
\hline TIPO DE COLUTORIOS & \multicolumn{1}{|c|}{ Media } & \multicolumn{1}{c|}{$\begin{array}{c}\mathrm{N}^{\circ} \\
\text { muestras }\end{array}$} & $\begin{array}{c}\text { Desv } \\
\text { estándar }\end{array}$ \\
\hline CETILPIRIDINIO & 113.67 & 15 & 110.52 \\
CONTROL & 1.93 & 15 & 3.17 \\
TRICLOSAN & 146.20 & 15 & 96.82 \\
Total & 87.27 & 45 & 103.81 \\
\hline
\end{tabular}


colutorio), se observa claramente en la Tabla 13, en el que la media aritmética de las variaciones fue significativamente mayor en el grupo de pacientes que recibieron triclosán (media $=146,200$ $\mathrm{UFC} / \mathrm{ml}$ ) que aquellos que recibieron cetilpiridinio (media $=113,670 \mathrm{UFC} / \mathrm{ml}$ ).

\section{Discusión}

De acuerdo con BERRIOS (2000) (4), ningún enjuague es $100 \%$ efectivo contra el crecimiento bacteriano; dicho investigador agrega que existe una eficacia moderada en la disminución del crecimiento con el uso de cloruro de cetilpiridinio y enjuagues que contengan triclosán. Nuestra investigación coincide con estos resultados, ya que ha mostrado que el uso de Triclosán y Cloruro de Cetilpiridinio reducen significativamente el recuento del número de colonias de streptococos de la cavidad oral; sin embargo en ningún caso se ha podido apreciar la ausencia de colonias. Esto puede explicarse por la naturaleza de dichos colutorios, los cuales en la mayoría de los casos producen una desnaturalización de las proteínas de las bacterias, lo cual no elimina la posibilidad posterior de un nuevo incremento en el crecimiento bacteriano. Es decir, que la naturaleza de los colutorios (llamados efectivamente "antisépticos") sólo cumplen la función de reducir en forma variada el crecimiento bacteriano.(3)

Lustosa de Castro (2001) (7) por otro lado demostró que la clorhexidina tiene mejor efecto que el cloruro de cetilpiridinio sobre la reducción de microorganismos microaerofílicos de la cavidad oral. Dichos gérmenes requieren de un ambiente de baja tensión de oxígeno, para crearse estas condiciones se requiere de mayor tiempo de permanencia de la bacteria en el lugar de crecimiento por lo que el uso de un colutorio que permanezca físicamente mayor tiempo sobre la superficie dentaria favorece las condiciones para mantener mayor ventaja sobre el crecimiento de dichos microorganismos. De igual manera en nuestra investigación el triclosán tuvo mayor eficacia que el Cloruro de cetilpiridinio en la reducción de streptococos orales, los cuales fueron cultivados en anaerobiosis; es decir en ausencia de oxígeno semejante al estudio de Lustosa por lo que puede hacerse una comparación con sus resultados. (7)

De otro lado, el mayor recuento de colonias está relacionado con el mayor número de superficies cariadas, como es demostrado por los resultados de MOTZLED (1994) (8) el cual, luego de realizar un recuento de $S$. Mutans, encontró una coincidencia entre los conteos de colonias y el riesgo cariogénico. El determinó que el porcentaje de muestra con mayor número de colonias equivalente al $31.2 \%$ pertenecía a los que tuvieron mayor prevalencia de caries; en nuestra investigación pudimos observar una relación similar, teniendo en cuenta que toda nuestra muestra perteneció a pacientes con alto riesgo de caries dental, el mayor número de superficies cariadas estuvo directamente relacionado con el recuento elevado de streptococos orales, si bien es cierto no se relaciona directamente con S. Mutans se tiene un parámetro de la relación entre streptococos orales y caries dental aunque el específico sea S. Mutans. $(1,2,8)$

Los resultados de este estudio son de suma importancia, pues ayudan a realizar una mejor selección del enjuague bucal a utilizar diariamente. Usar un buen enjuague bucal es imprescindible para evitar las enfermedades más prevalentes de la cavidad oral tales como gingivitis y caries dental. El enjuague bucal por si solo no resuelve el problema, el cuidado oral preventivo debe ser una combinación de varios factores 
que incluyen el uso de un buen dentífrico, el hilo dental, el enjuague bucal y visitas periódicas al odontólogo. Todos estos métodos de prevención tienen como propósito evitar la formación del biofilm oral. También es importante reducir la ingesta de sacarosa ya que favorece la acidogénesis de las bacterias y altera el $\mathrm{pH}$ de la boca afectando y desmineralizando el esmalte produciéndose como consecuencia la formación de caries dental. $(1,4,5,6)$

Basándonos en los resultados obtenidos en cuanto a la reducción del recuento de colonias de Streptococos orales de acuerdo a lo estipulado en la hipótesis del presente trabajo, podemos afirmar que los enjuagues bucales que contengan Triclosán en su composición como principio activo (adicionando a este el copolímero PVM/ MA al 2\%), tienen mayor eficacia antibacteriana contra streptococos orales, ya que se halló una asociación estadísticamente significativa entre el recuento final de colonias y el tipo de colutorio aplicado. Se observó que aquellos pacientes que realizaron enjuagues con triclosán presentaron que en el $60 \%$ de los casos se redujo a menos de 150,000 colonias post enjuague, un $33 \%$ presento un recuento de 150,000 a 300,000 y un porcentaje mínimo presentó recuentos mayores a 300,000 colonias, a diferencia de cloruro de cetilpiridinio, en el que el 53.3\% de los casos presentó recuento de colonias menores a 150,000 un $26.7 \%$ cuyos recuentos se encuentran entre 150,000 y 300,000 colonias y un $20 \%$ que presento un recuento de más de 300,000 colonias. Después de determinada la diferencia entre ambos recuentos de colonias para cada una de las muestras, se halló una mayor variación en el grupo de pacientes que usaron triclosán en el enjuague, esto se deduce de la mayor frecuencia de pacientes cuyos recuentos de colonias variaron, por lo tanto la media aritmética de las variaciones fue signifi- cativamente mayor en el grupo de pacientes que recibieron triclosán (media 146,200 colonias de S. orales) que aquellos que recibieron cloruro de cetilpiridinio (media 113,670 colonias de S. orales).

\section{Conclusiones}

1. La aplicación de colutorios de triclosán y cloruro de cetilpiridinio producen una reducción significativa en el recuento de colonias de streptococos de la cavidad oral.

2. Los pacientes que recibieron enjuagues con triclosán presentaron recuentos de colonias menores de 150,000 en el 60\% de los casos mientras que los pacientes que recibieron enjuagues con cloruro de cetilpiridinio presentaron recuentos de colonias menores de 150,000 en el 53.3\% de los casos.

3. Los pacientes que recibieron enjuagues con triclosán presentaron recuentos de colonias mayores a 300,000 en el 6.7\% de los casos mientras que aquellos pacientes que recibieron enjuagues con cloruro de cetilpiridinio presentaron recuentos de colonias mayores a 300,000 en el $20 \%$ de los casos.

4. El uso de colutorios de triclosán produjo una reducción media de 146,200 UFC/ $\mathrm{ml}$ de Streptococos y el uso de colutorios de cetilpiridinio produjo una reducción media de 113,670 UFC/ $\mathrm{ml}$ de Streptococos de la cavidad oral.

5. Se halló una reducción estadísticamente significativa en el recuento de colonias de streptococos en los pacientes tratados con algún tipo de colutorio que, los pacientes usados como controles.

6. El tipo de saliva guardó relación con el número de superficies de caries dental, es decir una saliva más viscosa y en menor cantidad 
es un factor que está relacionado con el mayor número de superficies cariadas.

7. Hubo asociación estadísticamente significativa entre el recuento inicial de colonias de streptococos (antes del enjuague) y el número de superficies de caries dental, a más superficies cariadas mayor recuento de colonias de streptococos orales.

\section{Referencias}

1. Abello R, Barrientos S. Generalidades sobre la caries dental. [Internet] Centro de Investigaciones Odontológicas 2000; Boletín N². Disponible en: http:/ / www.encolombia.com/odontologia/investigaciones/caries.htm

2. Balda R, Gonzales O. Evaluación del riesgo de caries dental como un proceso infeccioso. Acta Odontológica Venezolana 1999; 37(3) Edición Especial.

3. Bascones A, Mudarra S. Antisépticos en el tratamiento de la enfermedad periodontal. Avances en Periodoncia e Implantología 2002; 14(3).

4. Berrios R, Marrero J. El efecto de los enjuagadores bucales de mayor uso en las bacterias que causan la placa dental. Universidad de Puerto Rico, Recinto de Río Piedras, Facultad de Ciencias Naturales, Departamento de Matemáticas. [Internet]. 2001 [citado Junio 2002].Disponible en: http://www.eduesta.org/materiales/proyectos/Inv99-2000-II-7. pdf

5. Gaffar A, Nabi N. Recent advances in plaque, gingivitis, tartar and caries prevention technology. Int. Dent. J. 1994; 44, Supl.1.

6. Gultz J, Kaim J. An in vivo comparison of the antimicrobial activities of three mouthrinses. J Clin. Dent 1998; 9(2).

7. Lustosa De Castro S, Costa Lima M. Estudo in vivo da eficácia 2 antisépticos sobre microorganismos microaerofílicos da cavidade bucal. [Internet]. Revista dentística online 2001; Año1(2), Janeiro/Março. Disponible en: http:/ / www. ufsm.br/dentísticaonline

8. Moran J, Addy M, et al. A study to assess the plaque inhibitory activity of a new triclosan mouthrinse formulation. J Clin Periodontol. 2000; 27(11).

9. Motzfeld R, Linossier A. Prevalencia de caries y de Streptococcus Mutans en alumnos de $1^{\circ}$ año Facultad de Odontología de la Universidad de Chile. Rev. Fac. Odontol. Univ. Chile 1996; Vol.14(2) Julio/Diciembre.

10. Pineda M, Sotomayor J. Aplicación de métodos antisépticos previos al tratamiento odontológico para la reducción de la carga microbiana salival. Odontología Sanmarquina 2000; 1(5). Enero/Junio.

11. Porcel, M. “Una actitud activa en la atención odontológica. Incorporación de enjuagues antisépticos”. [Internet]. 2001 [citado Junio 2002]. Disponible en: http: / / www.red-dental.com/tranotas.htm

12. Stella Maimone. Triclosán y Gluconato de Clorhexidina. Visión 1998; 2(4).

Recibido: $14-11-12$

Aceptado: 28-12-12

Correspondencia: cecidont@yahoo.com 